\title{
BMJ Open Migrant perinatal depression study: a prospective cohort study of perinatal depression on the Thai-Myanmar border
}

\author{
Gracia Fellmeth, ${ }^{1,2}$ Emma H Plugge, ${ }^{3}$ Verena Carrara, ${ }^{2}$ Mina Fazel, ${ }^{4}$ May May Oo, ${ }^{2}$ \\ Yuwapha Phichitphadungtham, ${ }^{2}$ Mupawjay Pimanpanarak, ${ }^{2}$ Naw Kerry Wai, ${ }^{2}$ \\ Oh $\mathrm{Mu},{ }^{2}$ Prakaykaew Charunwatthana, ${ }^{5}$ François Nosten, ${ }^{2,3}$ Raymond Fitzpatrick, ${ }^{1}$ \\ Rose Mcgready ${ }^{2,3}$
}

To cite: Fellmeth $\mathrm{G}$, Plugge EH, Carrara V, et al. Migrant perinatal depression study: a prospective cohort study of perinatal depression on the ThaiMyanmar border. BMJ Open 2018;8:e017129. doi:10.1136/ bmjopen-2017-017129

- Prepublication history for this paper is available online. To view please visit the journal online (http://dx.doi.org/10. 1136/bmjopen-2017-017129).

Received 3 April 2017 Revised 6 September 2017 Accepted 7 September 2017

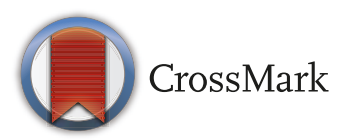

${ }^{1}$ Nuffield Department of Population Health, University of Oxford, Oxford, UK

${ }^{2}$ Faculty of Tropical Medicine,

Mahidol University, Shoklo

Malaria Research Unit, MahidolOxford Tropical Medicine

Research Unit, Mae Sot,

Thailand

${ }^{3}$ Nuffield Department of

Medicine, Centre for Tropical Medicine and Global Health,

University of Oxford, Oxford, UK

${ }^{4}$ Department of Psychiatry,

University of Oxford, Warneford

Hospital, Oxford, UK

${ }^{5}$ Department of Clinical Tropical

Medicine, Faculty of Tropical

Medicine, Mahidol University,

Bangkok, Thailand

Correspondence to

Dr Gracia Fellmeth;

gracia.fellmeth@dph.ox.ac.uk

\section{ABSTRACT}

Purpose Perinatal depression is a significant contributor to maternal morbidity. Migrant women in resource-poor settings may be at increased risk, yet little research has been conducted in low-income and middle-income settings. This prospective cohort study of migrant women on the Thai-Myanmar border aims to establish prevalence of perinatal depression, identify risk factors for perinatal depression and examine associations with infant outcomes.

Participants Participating women are labour migrants and refugees living on the Thai-Myanmar border. A total of 568 women were recruited in their first trimester of pregnancy and are being followed up to 1-year postpartum.

Findings to date At baseline, women in our study had a median age of 25 years, the predominant ethnicity was Sgaw Karen (48.9\%), agriculture was the main employment sector (39.2\%) and educational attainment was low with a median of 4 years of education. In the first trimester of pregnancy, a quarter $(25.8 \% ; 95 \% \mathrm{Cl} 22.3$ to 29.5) of all women were depressed as diagnosed by the Structured Clinical Interview for the Diagnosis of DSM-IV Disorders.

Future plans Follow-up is ongoing and expected to continue until January 2018. The prevalence of depression at later stages of pregnancy and during the first postpartum year will be identified, and associations between depression status and demographic, social, migration-related, medical, obstetric and infant factors will be quantified.

Trial registration number NCT02790905.

\section{INTRODUCTION}

Perinatal depression-a depressive episode occurring during pregnancy or up to 12 months postpartum-is a significant contributor to maternal morbidity. ${ }^{12}$ Globally, the burden falls disproportionately on those living in poverty. In high-income countries, the period prevalence of depression has been estimated at $18.4 \%$ in pregnancy and $19.2 \%$ postnatally. ${ }^{3}$ In low-income and middle-income countries (LMIC), prevalence estimates

\section{Strengths and limitations of this study}

- To our knowledge, this is the first prospective study of perinatal depression among migrant women in a low-income setting, and our study contributes significantly to the under-researched field of migrant mental health.

- This study will provide the first quantification of disease burden of perinatal depression and identification of associated factors on the ThaiMyanmar border.

- Findings will enable improved detection of perinatal depression as well as earlier and better management of affected women.

- Interviews were carried out by general clinicians rather than psychiatrists. This shortcoming was the result of an absence of psychiatric expertise in this setting. However, the use of generalists including local healthcare workers is a strength for the longterm sustainability of identifying and managing mental disorders in this population.

- There was a $26.1 \%$ loss to follow-up at the third trimester in this highly mobile population. Statistical analyses will be conducted to explore differences between those lost and those who completed the study.

are estimated at $25.3 \%$ in pregnancy and $19.0 \%$ postnatally. ${ }^{2}$ Point prevalence estimates from meta-analyses have found rates ranging from $7.4 \%$ to $12.8 \%$ in individual trimesters of pregnancy and a peak of $12.9 \%$ in the third month postpartum. ${ }^{34}$ However, these meta-analyses are limited to studies from high-income settings, and comparable estimates from LMIC are lacking.

The consequences of perinatal depression are significant. Affected women are at risk of chronic and recurrent depression, and the ability to work and provide care may be impaired. Depression in pregnancy has been linked to negative health behaviours such as substance misuse and poor uptake 
of antenatal care. ${ }^{3}$ Infants of depressed mothers are at increased risk of preterm birth, low birth weight, stunting in later childhood and poor neurodevelopmental and behavioural outcomes which may persist into adolescence and affect functional outcomes. ${ }^{5-7}$

Migrant women, whom we define as those who have left their place of origin regardless of circumstances, are at particular risk of perinatal depression. ${ }^{89}$ Stressors within their family, occupational and social circumstancesmany of which may have contributed to their decision to migrate-continue to impact on migrant populations in their place of settlement. ${ }^{89}$ Women who resettle within LMIC are at especially high risk. ${ }^{10}$ However, despite the bulk of global migration flows occurring in low-income and middle-income regions, the evidence on migrant mental health remains heavily skewed towards high-income destinations. A systematic review of perinatal mental disorders among migrant women identified 41 studies, of which 37 were conducted in high-income countries, four in middle-income countries and none in low-income countries. ${ }^{10}$ There is thus an urgent need for improved understanding and detection of perinatal depression in LMIC to enable quantification of the disease burden and effective management of the condition. $^{2}$

The Thai-Myanmar border area is home to an estimated 200000 labour migrants and 145000 refugees from Myanmar. ${ }^{11} 12$ The prevalence of perinatal depression within this setting has not previously been examined. A prospective cohort study of pregnant migrant women in this low-income setting was set up with the following objectives: (1) to determine the prevalence of perinatal depression; (2) to identify differences in prevalence at various stages of pregnancy and the postpartum period; (3) to identify demographic, social, medical and obstetric factors associated with perinatal depression; and (4) to examine associations between maternal depression and neurodevelopmental outcomes of infants. In this paper, we describe the design, recruitment and characteristics of the cohort.

\section{COHORT DESCRIPTION}

\section{Setting}

The study was carried out at Shoklo Malaria Research Unit (SMRU) in Mae Sot, Tak Province, Thailand. SMRU is a field station of the Mahidol-Oxford Tropical Medicine Research Unit, a research collaboration between Mahidol University (Thailand) and the University of Oxford (UK). SMRU has carried out research and provided maternity services on the Thai-Myanmar border area since 1986. Its clinics are located along the Thai side of the border, 30-60 km north and south of Mae Sot. Care is provided to refugee women and infants in Maela camp (MLA) and to rural labour migrants at Mawker Tai (MKT) and Wang Pha (WPA) (figure 1).
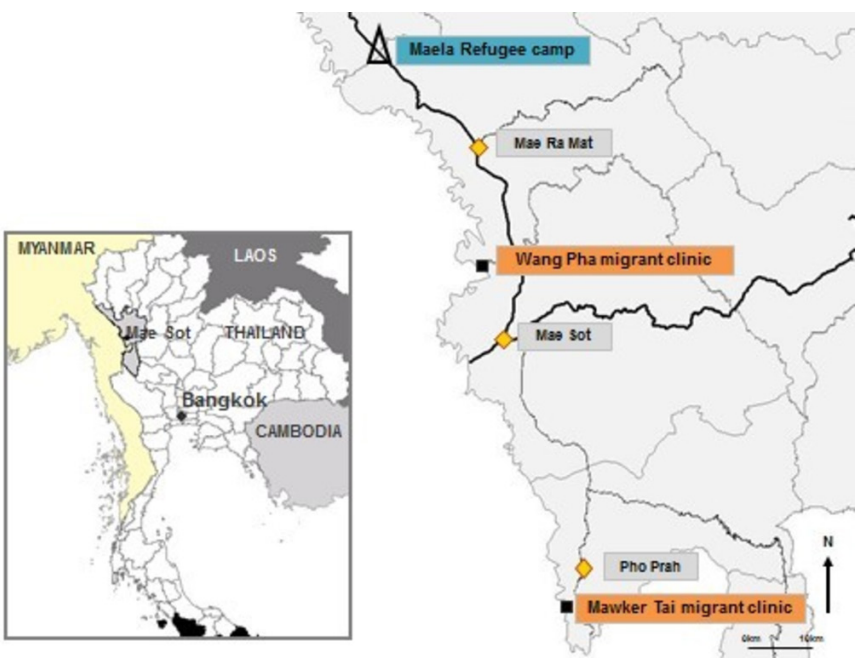

Figure 1 Map of study area showing refugee $(\Delta)$ and migrant clinics $(\square)$ (Credit: Dr Verena Carrara, Shoklo Malaria Research Unit).

\section{Population}

Refugees live in camps on the Thai side of the border. MLA is the largest refugee camp with a population of $37000 .{ }^{13}$ Within the refugee camp, non-governmental organisations provide healthcare, education, food rations and housing, and the United Nations High Commissioner for Refugees manages repatriation and resettlement programmes. ${ }^{13}$ Although the refugee camps provide a degree of security, opportunities for work and freedom of movement are severely limited. By contrast, labour migrants are a highly mobile population, residing in rural villages on both sides of the border and often many making daily commutes across the border for work. Labour migrants in this setting work predominantly in the agricultural sector and are paid minimal daily wages. ${ }^{11} 12$ Many labour migrants lack official documentation rendering them vulnerable to fines, arrest and deportation by the Thai authorities, and excluding them from accessing healthcare, social care and education. ${ }^{11}{ }^{12}$ In this paper, we use the term 'migrant' to include both refugee and labour migrant populations.

\section{Eligibility and recruitment}

This prospective cohort study includes first trimester pregnant migrant women attending SMRU antenatal clinics (ANC) at MLA, MKT and WPA. Women were eligible if they were aged 18 years or over, their estimated gestational age (EGA) as determined by ultrasound dating scan was less than 14 weeks, they had a viable pregnancy, planned to deliver at SMRU and were willing and able to participate. Eligible women were approached by a member of the study team while waiting to be seen at ANC. Recruitment took place between October 2015 and April 2016. Follow-up assessments will take place regularly until 12 months postpartum and will be complete in January 2018. Further follow-up is subject to funding. 


\section{Ethics and consent}

At recruitment, study staff provided eligible women with verbal and written explanations of the study. It was explained that participation was voluntary, that non-participation would not affect care and that consent could be withdrawn at any time. Women who agreed to participate provided consent in the form of a signature or thumbprint for those with low literacy. Participants are offered a small gift (of approximate value £1) at each visit and any travel costs incurred are reimbursed.

\section{Instruments}

Depression status is being ascertained using the depression items of the Structured Clinical Interview for the Diagnosis of DSM-IV Disorders (SCID), a widely used, semistructured diagnostic tool. ${ }^{14}$ The SCID was translated into Burmese and Sgaw Karen by two SMRU clinicians fluent in Burmese, Karen and English. Back-translation was carried out by two further SMRU clinicians who had not seen the original English version. Original and back-translated English versions were compared to ensure that semantic equivalence had been maintained. DSM-IV criteria were applied to SCID responses to establish diagnoses of Major Depressive Disorder, Minor Depressive Disorder and Depressive Disorder Not Otherwise Specified (NOS). The diagnostic category of Depressive Disorder NOS was included to capture the substantial proportion of women with symptoms of depression that were clinically significant but which did not meet the DSM-IV criteria for major or minor depression.

At inclusion in trimester one (T1), the Refugee Health Screener-15 (RHS-15) was also administered. The RHS-15 screens for psychological and somatic symptoms of depression, anxiety and post-traumatic stress disorder. ${ }^{15}$ The RHS-15 consists of 14 Likert-type response items and a distress thermometer that asks respondents to rate their distress on a visual scale of 1 to 10 . Burmese and Sgaw Karen versions of the RHS-15 were acquired from the RHS-15 authors. ${ }^{16}$

Data on demographic, social and migration data were collected using questionnaires. Medical and obstetric data will be obtained from participants' computerised medical records following delivery. Infant measurements including length and weight are being conducted using standardised instruments. Infant development is being assessed using the Shoklo Developmental Test, a locally developed neurological examination designed for field use in resource-constrained settings. ${ }^{17} 18$ The Shoklo Developmental Test has good correlation with the Griffiths Developmental Scales and has been used in our setting to evaluate the neurodevelopment of infants born to children with malaria in pregnancy and in ongoing studies of neonatal jaundice. ${ }^{17}$ 19-22 Mothers' developing relationships with their infants is being assessed with the Mother-to-Infant Bonding Scale. ${ }^{23}$

\section{Procedure}

A study timeline is shown in table 1. Data are being collected at eight time points: in the first (T1), second
(T2) and third (T3) trimesters of pregnancy and at 1 (T4), 3 (T5), 6 (T6), 9 (T7) and 12 (T8) months postpartum. Questionnaires and interviews are conducted by study staff in a private room in Sgaw Karen or Burmese according to women's preference. Verbal administration (rather than self-completion) is used due to low literacy rates within this population and limited comprehension of health-related written information, even among those able to read. ${ }^{24}$ SCID responses are independently scored by the study physician and an independent physician. Disagreements are resolved by discussion with a psychiatrist (MF). Women with depression are offered counselling and, when appropriate, antidepressant medication and follow-up at SMRU. Women with severe symptoms or active suicidal ideation are admitted for treatment and observation.

\section{Quality assurance and control}

The study team consists of SMRU physicians, midwives and counsellors. Midwives and counsellors are fluent in Burmese, Sgaw Karen and English and are themselves members of the local migrant community, and therefore sensitive to the needs of the population. Prior to recruitment, the study lead (GF) received training from the American Psychiatry Association in conducting SCID interviews. Counsellors and midwives underwent training in conducting interviews and counselling methods prior to the study. During the first month, all questionnaires and interviews were conducted with the study lead until counsellors and midwives were able to perform them unassisted. Thereafter, the study lead co-conducted interviews at one site per day to ensure quality.

\section{Sample size}

All women attending SMRU ANC in their first trimester of pregnancy during the recruitment period were invited to participate. Based on previous studies in this setting, we assumed a high participation rate and approximately $15 \%$ loss to follow-up. ${ }^{25}$ Our target sample size of 500 was based on an assumed approximate overall depression prevalence of $20 \%$ and $80 \%$ power (with two-sided $95 \%$ CIs) to detect associations of approximately 2.5-fold in magnitude and to run multiple regression analyses with up to four independent variables. ${ }^{26}$

\section{Data security and management}

All data are de-identified and entered into a password-protected Microsoft Excel database accessible only to SMRU study staff. Source questionnaires are held securely at SMRU ANC sites. Once follow-up is complete they will be stored at the SMRU head office.

\section{FINDINGS TO DATE}

Between October 2015 and April 2016, 627 eligible women attended SMRU ANC. Of these, 591 were approached and 568 (90.6\% of all eligible; $96.1 \%$ of those approached) women agreed to participate. Figure 2 shows the flow 


\begin{tabular}{|c|c|c|c|c|c|c|c|c|}
\hline \multirow[b]{2}{*}{ Time } & & \multicolumn{2}{|c|}{ Depression } & \multirow{2}{*}{$\begin{array}{l}\text { Demographic } \\
\text { and social }\end{array}$} & \multirow{2}{*}{$\begin{array}{l}\text { Medical } \\
\text { factors }\end{array}$} & \multirow{2}{*}{$\begin{array}{l}\text { Obstetric } \\
\text { factors }\end{array}$} & \multirow{2}{*}{$\begin{array}{l}\text { Infant } \\
\text { factors }\end{array}$} & \multirow{2}{*}{$\begin{array}{l}\text { Infant } \\
\text { bonding }\end{array}$} \\
\hline & & SCID & RHS-15* & & & & & \\
\hline \multicolumn{9}{|c|}{ Pregnancy } \\
\hline $\mathrm{T} 1$ & $\begin{array}{l}\text { First trimester } \\
(E G A<14)\end{array}$ & $x$ & Full & $x$ & & & & \\
\hline T2 & $\begin{array}{l}\text { Second trimester } \\
\text { (EGA 18-26) }\end{array}$ & $x$ & DT & & & & & \\
\hline T3 & $\begin{array}{l}\text { Third trimester (EGA } \\
28-38 \text { ) }\end{array}$ & $x$ & DT & $x$ & & & & \\
\hline \multicolumn{9}{|c|}{ Postpartum } \\
\hline T4 & $\begin{array}{l}\text { Onemonth } \\
\text { postpartum }\end{array}$ & $x$ & DT & & $x$ & $x$ & $x$ & $x$ \\
\hline T5 & $\begin{array}{l}\text { Three months } \\
\text { postpartum }\end{array}$ & & & & & & $x$ & \\
\hline T6 & $\begin{array}{l}\text { Six months } \\
\text { postpartum }\end{array}$ & $x$ & DT & & & & $x$ & $x$ \\
\hline $\mathrm{T} 7$ & $\begin{array}{l}\text { Nine months } \\
\text { postpartum }\end{array}$ & & & & & & $x$ & \\
\hline T8 & $\begin{array}{l}\text { Twelvemonths } \\
\text { postpartum }\end{array}$ & $x$ & DT & & $x$ & & $x$ & $x$ \\
\hline
\end{tabular}

*At T1, the full RHS-15 was administered. At subsequent visits, only the distress thermometer (DT) component of the RHS-15 was administered.

EGA, estimated gestational age; RHS-15, Refugee Health Screener-15; SCID, Structured Clinical Interview for the Diagnosis of DSM-IV Disorders.

of participants through the study from recruitment (T1) through to T3. Follow-up for T4 through T8 is still ongoing. Women who were eligible but missed due to language or staffing constraints did not differ significantly from those included by age, ethnicity or educational level. Of the 568 women who completed T1, $84.7 \%$ completed T2\% and $81.2 \%$ completed T3. Some women who did not attend at T2 returned at T3. The most common reason for participants not returning for follow-up was abortion in early pregnancy.

\section{Demographic characteristics}

At enrolment, the median age was 25 years (table 2) and mean EGA (SD) was 9.6 (2.3) weeks. Sgaw Karen was the predominant ethnicity and language among refugees, while Burman ethnicity and Burmese language were predominant among labour migrant women. The median years of education was 4 years, and almost half $(45.4 \%)$ of all participants had attended school for under 3 years. The main employment sector was agricultural work $(39.2 \%)$, although over a third of participants were not in paid employment $(35.7 \%)$.

\section{Depression status}

At baseline, the overall prevalence of depression as diagnosed by the SCID was $25.8 \%$ (table 3 ). There were significant differences in crude prevalence rates of depression between MKT and WPA, and between the migrant sites (MKT and WPA) combined and MLA. Explanations for these differences will be explored through regression analyses.

\section{STRENGTHS AND LIMITATIONS}

To our knowledge, this is the first prospective study of perinatal depression among migrant women in a resource-constrained setting. The active screening for depression will inform the early detection and treatment of this condition, enabling affected women to be supported and appropriate interventions to be developed. An improved understanding of the prevalence and risk factors of depression is a cornerstone to addressing the disease burden. Mental disorders are a neglected field in this and other low-income settings. The limited number of previous studies from this region have focused on specific subgroups including refugee children, ${ }^{27}$ Burmese political dissidents living in Bangkok, ${ }^{28}$ labour migrants workers in Mae $\mathrm{Sot}^{29}$ and Karenni refugees in northern Thailand. ${ }^{30}$ None have included pregnant or postpartum women. To our knowledge, this is also the first study to include both labour migrants and refugees, enabling direct comparison between these two distinct subgroups of the migrant population. As well as allowing the progression of depression through pregnancy and the postpartum period to be assessed, a significant strength of our cohort design is the collection of data on an extensive array of potential risk and associated factors including demographic, social, medical, obstetric and infant factors. 


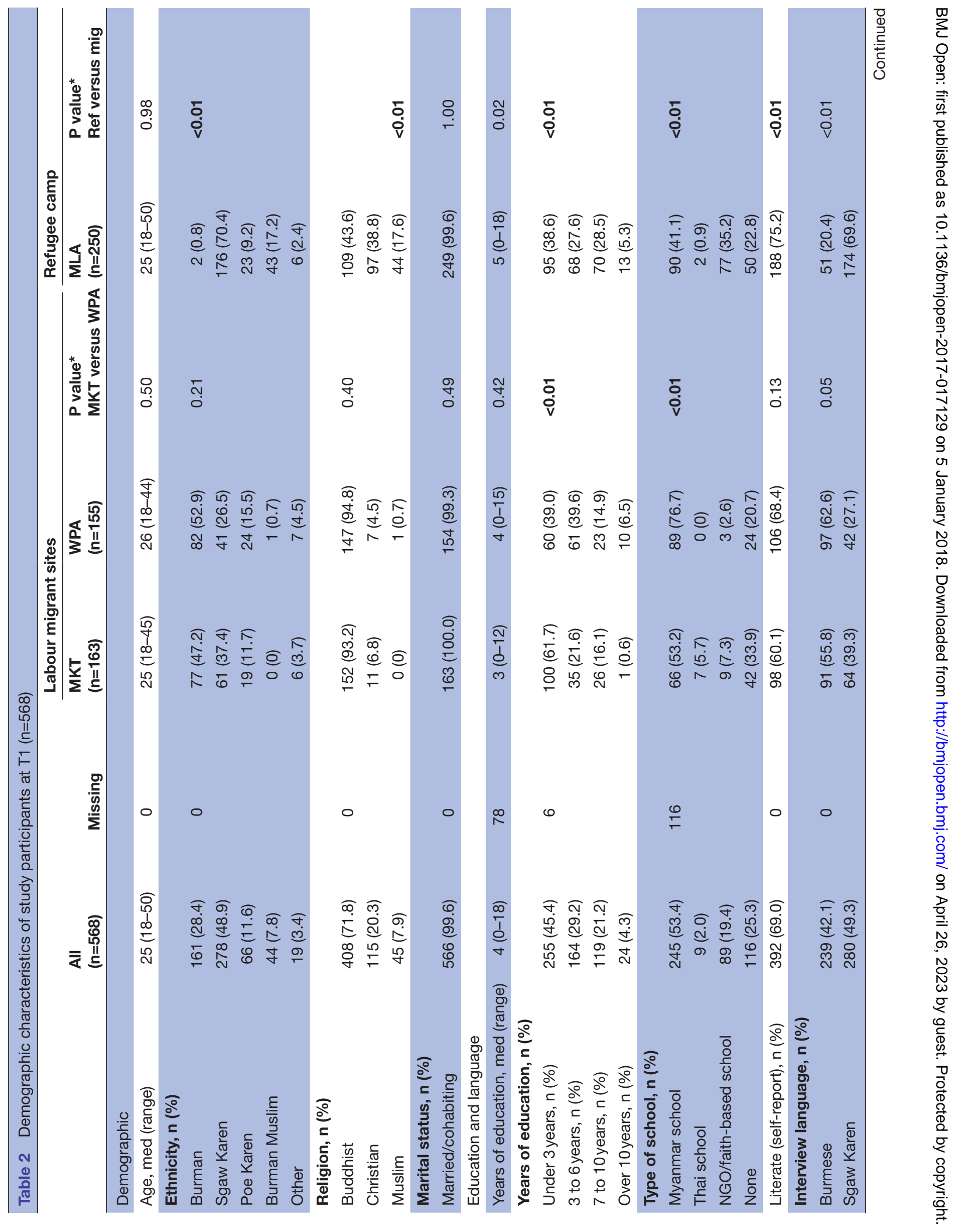




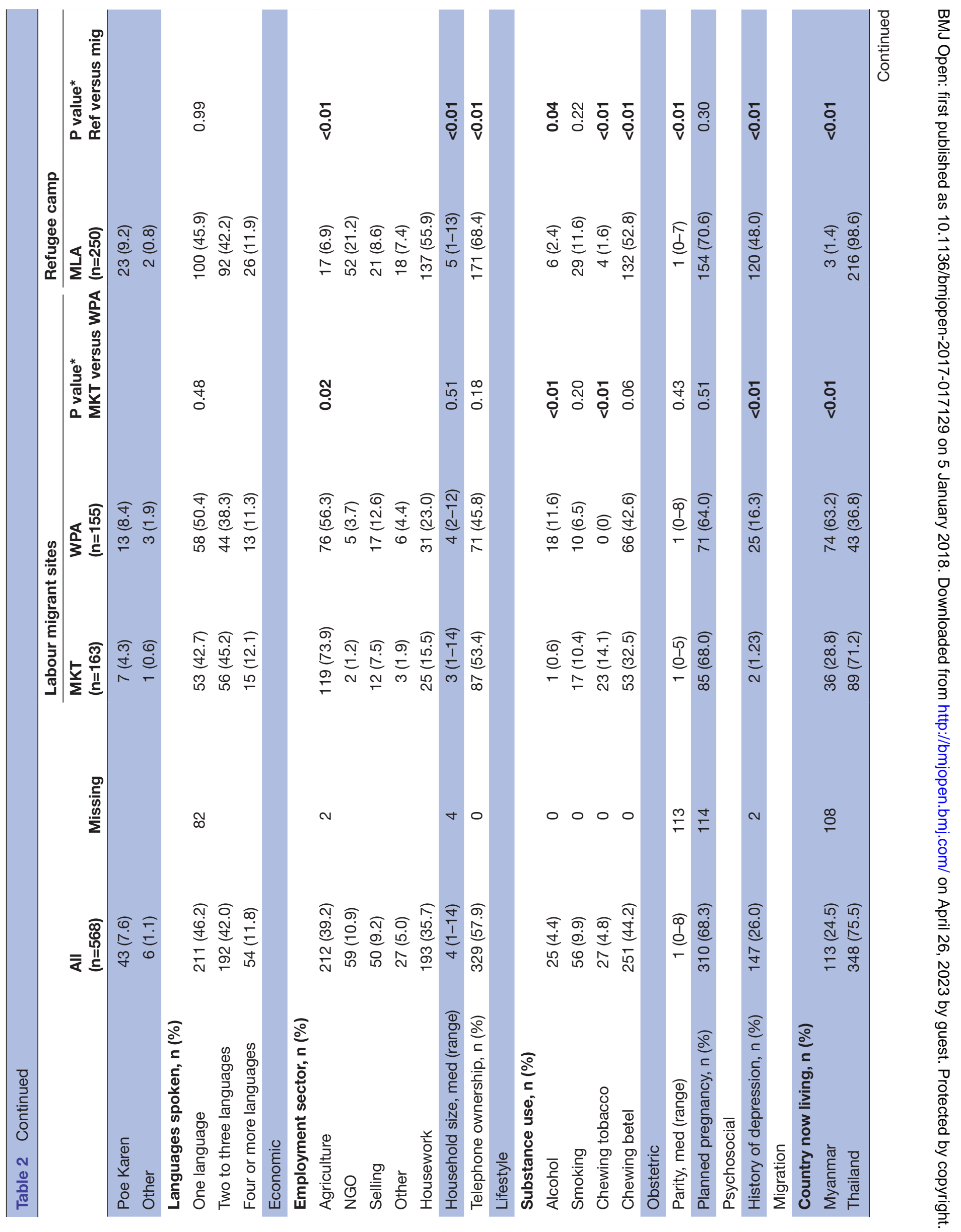




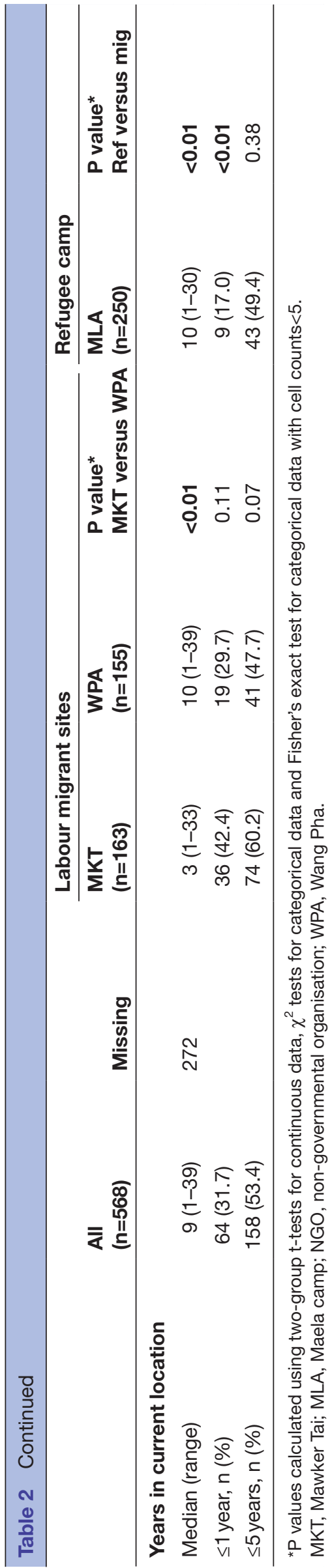

Overall, our study contributes to the under-researched field of migrant mental health from LMIC settings. ${ }^{10} 27$

A further strength of our study is that while most studies of mental disorders use screening tools to make mental state assessments, we used a diagnostic interview tool. ${ }^{10}$ Interviews were conducted by local healthcare staff who are themselves part of the local community. This enabled trust to be established with patients, and ensured high levels of cultural sensitivity. The fact that over $90 \%$ of women in this area attend ANC, coupled with our high response rate, means that our sample is representative of the general migrant population. ${ }^{25}$ The inclusion of the category of Depression NOS sheds light on an under-reported group of women who experience symptoms of depression that are clinically significant but do not quite meet the criteria for major or minor depression. In order to increase comparability to findings from other settings, our main statistical analyses will be limited to the more commonly reported categories of minor and major depression. We will also conduct additional analyses to explore the effects of including the NOS group.

There are also a number of limitations to our study. The absence of mental health expertise in our setting meant that it was not possible to obtain specialist psychiatry input. ${ }^{31} 32$ However, by providing experienced local healthcare workers with training in conducting interviews and counselling skills we maximised accuracy as much as possible. Furthermore, in a resource-constrained setting it is more appropriate in the long term for common mental disorders such as depression to be identified and managed by trained local workers, as specialist mental health professionals are rarely available. By training frontline healthcare staff to conduct these assessments and provide counselling, we ensured that our study promoted local capacity building, ownership, scalability and sustainability. ${ }^{33}$

A further limitation is that face-to-face administration of interviews may have resulted in a social desirability bias and a lower willingness to disclose sensitive information. ${ }^{34}$ Given the sensitive nature of many of the issues discussed, including depression, suicidal ideation and behaviour, intimate partner violence and trauma history, participants may have felt uncomfortable discussing these and under-reported relevant experiences, especially during a vulnerable period such as pregnancy. However, there is a strong oral tradition among the local population, and informal discussions are common and well accepted. ${ }^{35}$ We also believe that the sensitivity and local knowledge of the study staff helped to ensure that participants felt comfortable disclosing personal information.

The repeated administration of the SCID may have affected how women responded. We saw no evidence of questionnaire fatigue, perhaps because women in our setting attend clinic on a fortnightly basis through much of their pregnancy, and thus completing the SCID once per trimester was not perceived as burdensome. However, the repeated SCID interviews may have had a therapeutic effect by enabling participants an opportunity to talk 
Table 3 First trimester depression status among study participants by site and by migrant status

\begin{tabular}{|c|c|c|c|c|c|c|}
\hline & \multirow[b]{2}{*}{$\begin{array}{l}\text { All } \\
(n=568)\end{array}$} & \multicolumn{3}{|c|}{ Labour migrant sites } & \multicolumn{2}{|c|}{ Refugee camp } \\
\hline & & $\begin{array}{l}\text { MKT } \\
(n=163)\end{array}$ & $\begin{array}{l}\text { WPA } \\
(n=155)\end{array}$ & $\begin{array}{l}p \text { Value } \\
\text { MKT versus } \\
\text { WPA }\end{array}$ & $\begin{array}{l}\text { MLA } \\
(n=250)\end{array}$ & $\begin{array}{l}\text { p Value } \\
\text { Ref versus mig }\end{array}$ \\
\hline Any depression & $146(25.8)$ & $29(17.9)$ & $46(29.7)$ & 0.01 & $71(28.4)$ & 0.03 \\
\hline Major depression & $9(1.6)$ & $0(0)$ & $2(1.3)$ & & $7(2.8)$ & \\
\hline Minor depression & $37(6.5)$ & $6(3.7)$ & 19 (12.3) & & $12(4.8)$ & \\
\hline Depression NOS & $100(17.6)$ & 23 (14.2) & 25 (16.1) & & $52(20.8)$ & \\
\hline Negative & 421 (74.2) & $133(82.1)$ & 109 (70.3) & & $179(71.6)$ & \\
\hline
\end{tabular}

MKT, Mawker Tai; MLA, Maela camp; NOS, Not Otherwise Specified; WPA, Wang Pha.

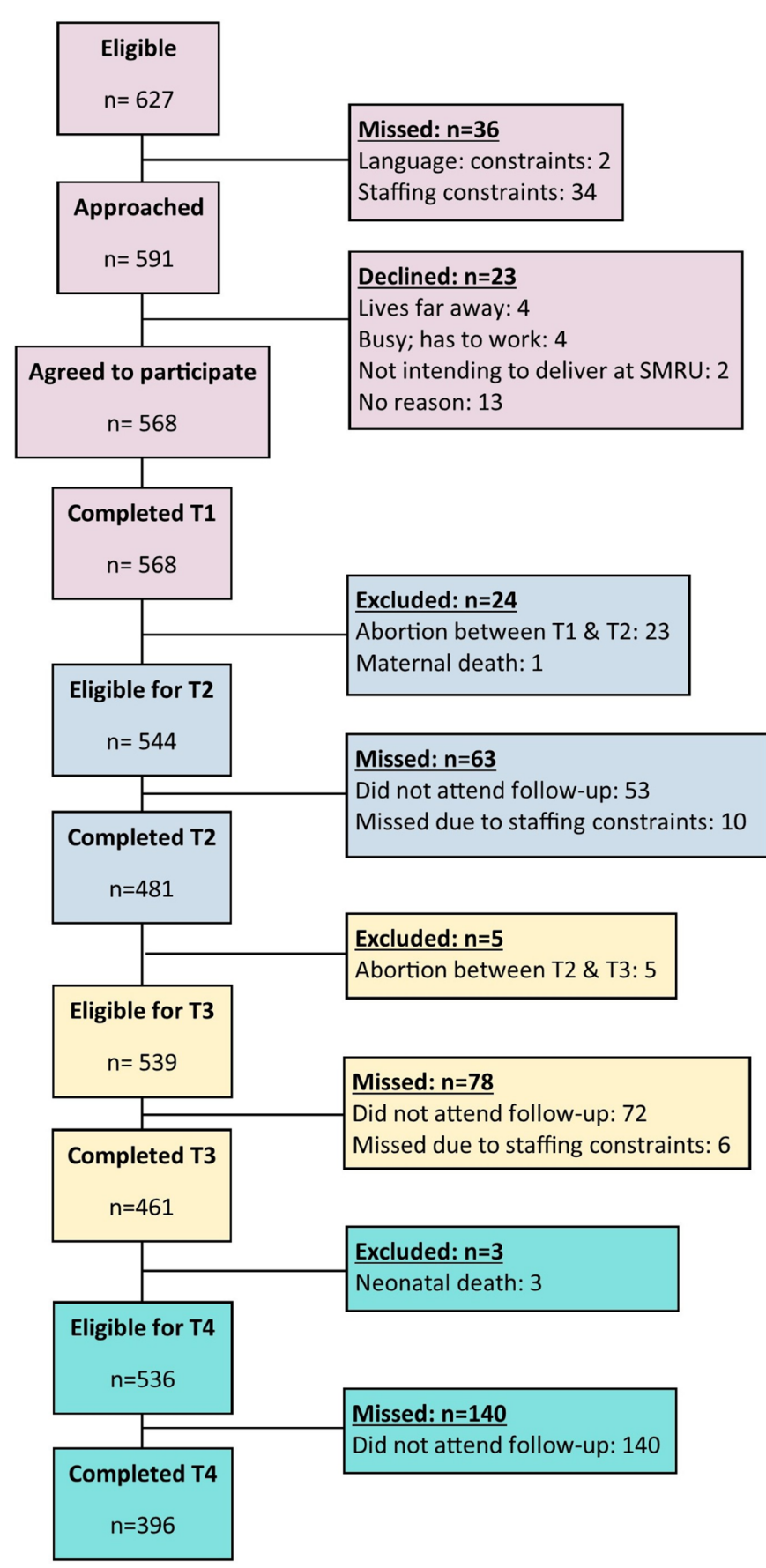

Figure 2 Flow of participants through study from recruitment to T3. SMRU, Shoklo Malaria Research Unit. and share any worries. This possibility will be taken into account in the interpretation of prevalence of depression after the baseline assessment.

Infants' neurodevelopmental outcomes will need to be interpreted with caution as assessments within the first year of life may not be sensitive enough to identify subtle differences between infants. Ideally, the cohort of infants would be followed up longer term. Nevertheless, it may be possible by 12 months to see trends in the progression of global development. The use of a more widely used tool such as the Bayley Scales would have been preferable. However, staffing and resource constraints, the length of the full Bayley test and a number of test items being difficult to convey in the local cultural context meant this was not possible. ${ }^{17} 18$ The Shoklo Developmental Test has been used extensively in our setting and its strong correlation with the Griffiths Developmental Scale-a standardised neurodevelopmental assessment tool-gives confidence to the results. ${ }^{1718}$ Should further follow-up become possible, it would be important to consider a wider range of validated instruments to test child outcomes.

Finally, our overall loss to follow-up to date of approximately $20 \%$ is higher than our anticipated loss of $15 \%$. This figure may increase in subsequent postpartum waves. We plan to explore differences between those included and lost in our analyses in order to assess potential implications for generalisability of results.

\section{CONCLUSION}

Addressing perinatal depression among migrant communities in LMIC is necessary to promote maternal mental health and address key sustainable development goals including ensuring good health and wellbeing, establishing gender equality and reducing global inequalities both within and among countries. Establishing the prevalence of and risk factors for perinatal depression among migrant women on the ThaiMyanmar border will enable the burden of disease to be quantified, and earlier, more effective identification and management of affected women. We expect that observations and recommendations arising from this study will be of importance and relevance to other LMIC settings. 
Acknowledgements We wish to thank Dr Mary Ellen G Tyrovoutis, Dr Bert van Enter and Dr Aye Min for overseeing the study at Wang Pha, Mawker Tai and Maela respectively; Suphak Nosten for her insight and guidance into Karen and Burmese culture; Jacher Wiladphaingern for his support in data management; and all the women who participated in this study for their time, patience and trust.

Contributors Conceptualisation of the study: GF, RM, VC, EP, MF, RF, PC, FN. Data curation: MMO, YP, MP, NKW, OM, GF, VC, RM. Analysis: GF, RM, EP, VC. Write-up: all authors contributed to the writing of the manuscript.

Funding SMRU is part of the Wellcome Trust Mahidol University Oxford Tropical Medicine Research Program funded by the Wellcome Trust (WT-106698). This article is compliant with the funding body's open access policy. The funding body had no role in the design, collection, analysis or interpretation of data, nor in the writing or submission of the manuscript for publication.

\section{Competing interests None declared.}

Ethics approval Ethics approval was granted by the University of Oxford Tropical Research Ethics Committee (OxTREC 28-15), Mahidol University Faculty of Tropical Medicine Ethics Committee (TMEC 15-045) and the Tak Border Community Advisory Board (T-CAB 6/2/2015).

Provenance and peer review Not commissioned; externally peer reviewed.

Data sharing statement The data are not available freely. However, we welcome specific and detailed proposals for collaboration. Enquiries and requests for further information should be made to corresponding author.

Open Access This is an Open Access article distributed in accordance with the terms of the Creative Commons Attribution (CC BY 4.0) license, which permits others to distribute, remix, adapt and build upon this work, for commercial use, provided the original work is properly cited. See: http://creativecommons.org/ licenses/by/4.0/

(c) Article author(s) (or their employer(s) unless otherwise stated in the text of the article) 2018. All rights reserved. No commercial use is permitted unless otherwise expressly granted.

\section{REFERENCES}

1. Howard LM, Molyneaux E, Dennis CL, et al. Non-psychotic mental disorders in the perinatal period. Lancet 2014;384:1775-88.

2. Gelaye B, Rondon MB, Araya R, et al. Epidemiology of maternal depression, risk factors, and child outcomes in low-income and middle-income countries. Lancet Psychiatry 2016;3:973-82.

3. Gavin NI, Gaynes BN, Lohr KN, et al. Perinatal depression: a systematic review of prevalence and incidence. Obstet Gynecol 2005;106(5 Pt 1):1071-83.

4. Bennett HA, Einarson A, Taddio A, et al. Prevalence of depression during pregnancy: systematic review. Obstet Gynecol 2004;103:698-709.

5. Stein A, Pearson RM, Goodman SH, et al. Effects of perinatal mental disorders on the fetus and child. Lancet 2014;384:1800-19.

6. Grigoriadis S, VonderPorten EH, Mamisashvili L, et al. The impact of maternal depression during pregnancy on perinatal outcomes: a systematic review and meta-analysis. J Clin Psychiatry 2013;74:e321-41.

7. Herba CM, Glover V, Ramchandani PG, et al. Maternal depression and mental health in early childhood: an examination of underlying mechanisms in low-income and middle-income countries. Lancet Psychiatry 2016;3:983-92.

8. Collins $\mathrm{CH}$, Zimmerman C, Howard LM. Refugee, asylum seeker, immigrant women and postnatal depression: rates and risk factors. Arch Womens Ment Health 2011;14:3-11.

9. IOM. Glossary on Migration. 25 ed. Geneva: International Organization for Migration, 2011.

10. Fellmeth G, Fazel M, Plugge E. Migration and perinatal mental health in women from low- and middle-income countries: a systematic review and meta-analysis. BJOG 2017;124:742-52.

11. Pearson R, Kusakabe K. Thailand's hidden workforce: Burmese migrant women factory workers. London: Zed Books, 2012.

12. Dunlop N. Invisible people: stories of migrant labourers in Thailand. Bangkok: Raks Thai Foundation, 2011.
13. TBC The Border Consortium. Annual Report 2016. 2016.

14. Spitzer RL, Williams JB, Gibbon M, et al. The structured clinical interview for dsm-iii-r (scid). I: History, rationale, and description. Arch Gen Psychiatry 1992;49:624-9.

15. Hollifield M, Verbillis-Kolp S, Farmer B, et al. The refugee health screener-15 (rhs-15): Development and validation of an instrument for anxiety, depression, and ptsd in refugees. Gen Hosp Psychiatry 2013;35:202-9.

16. Pathways to Wellness. Introductory and referral scripts for the RHS-15 Refugee Health Technical Assistance Center: http:// refugeehealthta.org/wp-content/uploads/2012/09/RHS15_Packet_ PathwaysToWellness-1.pdf. 2012. [The RHS-15 is a tool for screening refugees for emotional distress and mental health. Packet includes the RHS- tool, background on the tool's development, and guidelines on using the RHS- with recently resettled refugees. RHShas also been translated into Somali, Russian, Arabic, Burmese, Karen, and Nepali.] http://www.lcsnw.org/pathways/index.html.

17. Haataja L, McGready R, Arunjerdja R, et al. A new approach for neurological evaluation of infants in resource-poor settings. Ann Trop Paediatr 2002;22:355-68.

18. McGready R, Simpson J, Panyavudhikrai S, et al. Neonatal neurological testing in resource-poor settings. Ann Trop Paediatr 2000;20:323-36.

19. McGready R, Ashley EA, Moo E, et al. A randomized comparison of artesunate-atovaquone-proguanil versus quinine in treatment for uncomplicated falciparum malaria during pregnancy. J Infect Dis 2005;192:846-53.

20. McGready R, Tan SO, Ashley EA, et al. A randomised controlled trial of artemether-lumefantrine versus artesunate for uncomplicated plasmodium falciparum treatment in pregnancy. PLoS Med 2008;5:e253.

21. Villegas L, McGready R, Htway M, et al. Chloroquine prophylaxis against vivax malaria in pregnancy: a randomized, double-blind, placebo-controlled trial. Trop Med Int Health 2007;12:209-18.

22. Griffiths R. The Griffiths Mental Development Scales. Henley: Association for Research in Infant and Child Development, Test Agency, 1996.

23. Taylor A, Atkins R, Kumar R, et al. A new Mother-to-Infant Bonding Scale: links with early maternal mood. Arch Womens Ment Health 2005;8:45-51.

24. Carrara VI, Hogan C, De Pree C, et al. Improved pregnancy outcome in refugees and migrants despite low literacy on the Thai-Burmese border: results of three cross-sectional surveys. BMC Pregnancy Childbirth 2011;11:45.

25. Thielemans L, Trip-Hoving M, Bancone G, et al. Neonatal Hyperbilirubinemia in a Marginalized Population on the ThaiMyanmar Border: a study protocol. BMC Pediatr 2017;17:32.

26. Tabachnick BG, Fidell LS. Using multivariate statistics. 6th edition. London: Pearson, 2013.

27. Meyer S, Murray LK, Puffer ES, et al. The nature and impact of chronic stressors on refugee children in Ban Mai Nai Soi camp, Thailand. Glob Public Health 2013;8:1027-47.

28. Allden K, Poole $\mathrm{C}$, Chantavanich $\mathrm{S}$, et al. Burmese political dissidents in Thailand: trauma and survival among young adults in exile. Am J Public Health 1996;86:1561-9.

29. Meyer SR, Decker MR, Tol WA, et al. Workplace and security stressors and mental health among migrant workers on the Thailand-Myanmar border. Soc Psychiatry Psychiatr Epidemiol 2016;51:713-23.

30. Lopes Cardozo B, Talley L, Burton A, et al. Karenni refugees living in Thai-Burmese border camps: traumatic experiences, mental health outcomes, and social functioning. Soc Sci Med 2004;58:2637-44.

31. Zaw KM. Psychiatric services in Myanmar: a historical perspective. Psychiatric Bulletin 1997;21:506-9.

32. Office W, MoH M. WHO-AIMS Report on Mental Health Systems in Myanmar. Myanmar: World Health Organization, 2006. http://www. who.int/mental_health/evidence/myanmar_who_aims_report.pdf.

33. Marley JV, Kotz J, Engelke C, et al. Validity and Acceptability of Kimberley Mum's Mood Scale to Screen for Perinatal Anxiety and Depression in Remote Aboriginal Health Care Settings. PLoS One 2017;12:e0168969.

34. Bowling A. Mode of questionnaire administration can have serious effects on data quality. J Public Health 2005;27:281-91.

35. Delang C. Living at the edge of Thai society: the Karen in the highlands of Northern Thailand. London: Routledge Curzon, 2003. 